Christmann, Bernd; Schwerdt, Daniel; Wazlawik, Martin

\title{
Perspektiven auf sexualisierte Gewalt in schulischen Kontexten. Konzepte, Befunde, Perspektiven
}

Die deutsche Schule 111 (2019) 2, S. 230-242

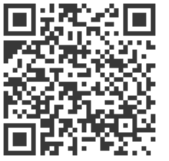

Quellenangabe/ Reference:

Christmann, Bernd; Schwerdt, Daniel; Wazlawik, Martin: Perspektiven auf sexualisierte Gewalt in schulischen Kontexten. Konzepte, Befunde, Perspektiven - In: Die deutsche Schule 111 (2019) 2, S. 230-242 - URN: urn:nbn:de:0111-pedocs-201585 - DOI: 10.25656/01:20158

https://nbn-resolving.org/urn:nbn:de:0111-pedocs-201585

https://doi.org/10.25656/01:20158

in Kooperation mit / in cooperation with:

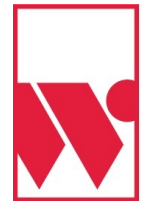

\section{WAXMANN}

www.waxmann.com

http://www.waxmann.com

\section{Nutzungsbedingungen}

Gewährt wird ein nicht exklusives, nicht übertragbares, persönliches und beschränktes Recht auf Nutzung dieses Dokuments. Dieses Dokument ist ausschließlich für den persönlichen, nicht-kommerziellen Gebrauch bestimmt. Die Nutzung stellt keine Übertragung des Eigentumsrechts an diesem Dokument dar und gilt vorbehaltlich der folgenden Einschränkungen: Auf sämtlichen Kopien dieses Dokuments müssen alle Urheberrechtshinweise und sonstigen Hinweise auf gesetzlichen Schutz beibehalten werden. Sie dürfen dieses Dokument nicht in irgendeiner Weise abändern, noch dürfen Sie dieses Dokument für öffentliche oder kommerzielle Zwecke vervielfältigen, öffentlich ausstellen, aufführen, vertreiben oder anderweitig nutzen.

Mit der Verwendung dieses Dokuments erkennen Sie die Nutzungsbedingungen an.

\section{Terms of use}

We grant a non-exclusive, non-transferable, individual and limited right to using this document.

This document is solely intended for your personal, non-commercial use. Use of this document does not include any transfer of property rights and it is conditional to the following limitations: All of the copies of this documents must retain all copyright information and other information regarding legal protection. You are not allowed to alter this document in any way, to copy it for public or commercial purposes, to exhibit the document in public, to perform, distribute or otherwise use the document in public.

By using this particular document, you accept the above-stated conditions of use.

\section{Kontakt / Contact:}

peDOCS

DIPF | Leibniz-Institut für Bildungsforschung und Bildungsinformation

Informationszentrum (IZ) Bildung

E-Mail: pedocs@dipf.de

Internet: www.pedocs.de

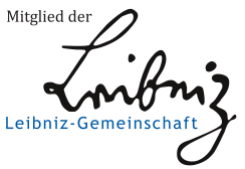




\section{Die Zeitschrift für \\ Deutsche Erziehungswissenschaft

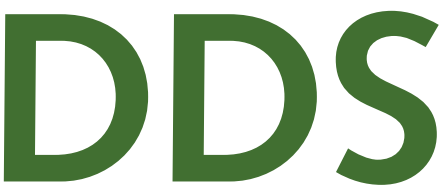 Schule Bildungspolitik und pädagogische Praxis}

\section{Herausforderungen und Perspektiven der Lehrkräftequalifizierung}

2019 R. Porsch

Berufswahlüberprüfung im Lehramtsstudium

P. Tulowitzki, I. Hinzen \& M. Roller

Qualifizierung von Schulleiter*innen in Deutschland

B. Fritzsche, S. Schuler \& G. Wittmann

Das berufliche Selbstverständnis von Multiplikator*innen für den Mathematikunterricht an Grundschulen

S. Niehoff, K. Fussangel, W.-D. Lettau \& F. Radisch Individuelle Förderung in der Ganztagsgrundschule

G. Graßhoff, C. Haude, T.-S. Idel, C. Bebek \& A. Schütz

Die Eigenlogik des Nachmittags

Berichte und Diskussion zum Schwerpunktthema

A. A. Wojciechowicz \& M. Vock

Wiedereinstieg in den Lehrerberuf nach der Flucht

B. Christmann, D. Schwerdt \& M. Wazlawik

Perspektiven auf sexualisierte Gewalt in schulischen Kontexten

M. Heinrich, G. Wolfswinkler, I. van Ackeren, N. Bremm \& L. Streblow Multiparadigmatische Lehrerbildung 


\section{Die Deutsche Schule Zeitschrift für Erziehungswissenschaft, Bildungspolitik und pädagogische Praxis}

Herausgeber: Gewerkschaft Erziehung und Wissenschaft im DGB in Zusammenarbeit mit der Max-Traeger-Stiftung

Redaktion: Prof. Dr. Isabell van Ackeren (Essen), Dr. Götz Bieber (Ludwigsfelde), Prof. Dr. Kathrin Dedering (Erfurt), Detlef Fickermann (Kamen), Prof. Dr. Martin Heinrich (Bielefeld), Prof. Dr. Marianne Krüger-Potratz (Münster)

Geschäftsführerin: Sylvia Schütze, Universität Bielefeld, Fakultät für Erziehungswissenschaft, AG 4, Universitätsstraße 25, 33615 Bielefeld, E-Mail: redaktion@dds-home.de Vorsitzende der Redaktion: Prof. Dr. Isabell van Ackeren (Essen)

Beirat: Prof. Dr. Herbert Altrichter (Linz-Auhof), Dr. Christine Biermann (Bielefeld), Marianne Demmer (Wilnsdorf), Prof. Dr. Mats Ekholm (Karlstad), Prof. Dr. Hans-Peter Füssel (Berlin), Prof. Dr. Friederike Heinzel (Kassel), Prof. Dr. Thomas Höhne (Hamburg), Prof. Dr. Klaus Klemm (Essen), Prof. Dr. Eckhard Klieme (Frankfurt a.M.), Prof. Dr. Katharina Maag Merki (Zürich), Prof. Dr. Heinrich Mintrop (Berkeley), Prof. Dr. Angelika Paseka (Hamburg), Prof. Dr. Nicolle Pfaff(Essen), Hermann Rademacker (München), Prof. Dr. Sabine Reh (Berlin), Prof. Dr. Hans-Günter Rolff (Dortmund), Prof. Andreas Schleicher (Paris), Dr. Gundel Schümer (Berlin), Jochen Schweitzer (Münster), Prof. Dr. Knut Schwippert (Hamburg), Ulrich Steffens (Wiesbaden), Prof. Dr. Klaus-Jürgen Tillmann (Berlin)

Beitragseinreichung und Double-blind Peer Review: Manuskripte (nur Originalbeiträge) werden als Word-Datei an die Geschäftsführung (redaktion@dds-home.de) erbeten. Bitte beachten Sie die Hinweise zur Manuskriptgestaltung (www.dds-home.de). Seit dem 103. Jahrgang (2011) durchlaufen alle Fachartikel in der DDS (Texte zum Themenschwerpunkt und für die Rubrik „Weitere Beiträge“) ein externes Review-Verfahren. Nach einer redaktionellen Prüfung der eingereichten Aufsätze im Hinblick auf ihre grundsätzliche Eignung für die DDS schließt sich eine Begutachtung im Doppelblindverfahren durch ehrenamtlich tätige Gutachter*innen an.

Die Deutsche Schule erscheint vierteljährlich. Zusätzlich zu den vier Heften pro Jahrgang können Beihefte erscheinen. Unter www.waxmann.com und www.dds-home.de finden Sie weitere Informationen. Die DDS ist indiziert in ESCI, FIS Bildung und Proquest und für weitere Indizierungen vorgeschlagen.

Preise und Bezugsbedingungen: Jahresabonnement 59,00 €, für GEW-Mitglieder/Studierende $43,00 €$, inkl. Online-Zugang für Privatpersonen. Campuslizenz auf Anfrage. Die Preise verstehen sich zzgl. Versandkosten. Ein Einzelheft kostet 18,00€ inkl. Versandkosten. Abbestellungen spätestens 6 Wochen vor Ablauf des Jahresabonnements.

ISSN 0012-0731

(C) Waxmann Verlag GmbH, 2019

Steinfurter Straße 555, 48159 Münster, Telefon: 02 51/2 6504 0, Fax: 02 51/2 6504 26,

Internet: www.waxmann.com, E-Mail: info@waxmann.com

Anzeigenverwaltung: Waxmann Verlag GmbH, Paula Brauer: brauer@waxmann.com Druck: mediaprint solutions GmbH, Paderborn

Satz: Stoddart Satz- und Layoutservice, Münster

Die Zeitschrift und alle in ihr enthaltenen Beiträge und Abbildungen sind urheberrechtlich geschützt. Jede Verwendung außerhalb der engen Grenzen des Urheberrechtsgesetzes ist ohne Zustimmung des Verlags unzulässig und strafbar. Unter dieses Verbot fallen insbesondere die gewerbliche Vervielfältigung per Kopie, die Aufnahme in elektronische Datenbanken und die Vervielfältigung auf CD-Rom und allen anderen elektronischen Datenträgern. 
DDS - Die Deutsche Schule Zeitschrift für Erziehungswissenschaft, Bildungspolitik und pädagogische Praxis https://doi.org/10.31244/dds.2019.02

111. Jahrgang 2019 / Heft 2

\section{INHALT}

NACHRUF

Trauer um Prof. Dr. Manfred Weiß 125

\section{EDITORIAL}

Martin Heinrich \& Isabell van Ackeren

Editorial zum Schwerpunktthema: Herausforderungen und

Perspektiven der Lehrkräftequalifizierung.

\section{HERAUSFORDERUNGEN UND PERSPEKTIVEN DER LEHRKRÄFTEQUALIFIZIERUNG}

\section{Raphaela Porsch}

Berufswahlüberprüfung in Praxisphasen im Lehramtsstudium: unvermeidbar und ergebnisoffen

Befunde einer Längsschnittuntersuchung

Pierre Tulowitzki, Imke Hinzen \& Marvin Roller

Die Qualifizierung von Schulleiter*innen in Deutschland ein bundesweiter Überblick

Bettina Fritzsche, Stephanie Schuler \& Gerald Wittmann

Das berufliche Selbstverständnis von Multiplikatorinnen für den

Mathematikunterricht an Grundschulen.

Stephanie Niehoff, Kathrin Fussangel, Wolf-Dieter Lettau \& Falk Radisch

Individuelle Förderung in der Ganztagsgrundschule ein Anlass zur Kooperation?

Gunther Graßhoff, Christin Haude, Till-Sebastian Idel, Carolin Bebek \& Anna Schütz

Die Eigenlogik des Nachmittags

Explorative Beobachtungen aus Ethnografien

$\mathrm{zu}$ außerunterrichtlichen Angeboten 


\section{BERICHTE ZUM SCHWERPUNKTTHEMA}

Anna Aleksandra Wojciechowicz \& Miriam Vock

Wiedereinstieg in den Lehrerberuf nach der Flucht

mit dem Refugee Teachers Program in Brandenburg.

Bernd Christmann, Daniel Schwerdt \& Martin Wazlawik

Perspektiven auf sexualisierte Gewalt in schulischen Kontexten:

Konzepte, Befunde, Perspektiven

\section{DISKUSSION ZUM SCHWERPUNKTTHEMA}

Martin Heinrich, Günther Wolfswinkler, Isabell van Ackeren,

Nina Bremm \& Lilian Streblow

Multiparadigmatische Lehrerbildung

Produktive Auswege aus dem Paradigmenstreit?

Die Zeitschrift für

Deutsche Erziehungswissenschaft

DDS Schule Bildungspolitik und pädagogische Praxis Herausgegeben von der Gewerkschaft Erziehung und Wissenschaft

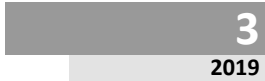

\section{Vorschau}

Themenschwerpunkt: Wissenstransfer und Implementation

Wissenstransfer und Implementation sind zentrale Topoi der Schulentwicklung. Zugleich wird der Begriff des „Wissenstransfers“ zunehmend ambivalent, angesichts der sich häufenden Problemdiagnosen mit Blick auf die erfolgreiche Implementierung empirisch gesicherten Wissens in Schulentwicklungsbemühungen. Wissenstransfer erfolgt nicht als Übermittlung eines festen Wissensbestands von Kontext A nach Kontext B. Prozesse der Adaption, der Aneignung, der "Re-Kontextualisierung" (Fend) oder des „Nacherfindens" (Kussau) erscheinen notwendig. Systematische empirische Forschung zu Entwicklungsprojekten ist allerdings rar. Das Heft versucht, diesen Diskurs zu beleben.

Heft 3/2019 der DDS erscheint im September 2019.

Waxmann • Steinfurter Str. $555 \bullet 48159$ Münster • www.waxmann.com 
Bernd Christmann, Daniel Schwerdt \& Martin Wazlawik

\title{
Perspektiven auf sexualisierte Gewalt in schulischen Kontexten: Konzepte, Befunde, Perspektiven
}

\section{Zusammenfassung}

Die Forschung zu sexualisierter Gewalt hat sich zuletzt dynamisch entwickelt. Dadurch eröffnen sich neue Perspektiven auf schulische Kontexte. Aktuelle Forschungsprojekte erweitern das hier vorhandene Wissen über die Prävalenz von sexualisierter Gewalt, präsentieren neue Präventionskonzepte, verdeutlichen den Qualifizierungsbedarf von Lehrkräften und zeigen Herausforderungen und Chancen bei der Entwicklung institutioneller Schutzkonzepte auf. Es werden jedoch auch neue Desiderate offenkundig. Schlüsselwörter: sexualisierte Gewalt, Schule, Prävention, Intervention

\section{Perspectives on Sexual Violence in School Contexts: Concepts, Findings, Prospects}

\begin{abstract}
Research on sexual violence has developed dynamically in recent years. This opens up new perspectives on school contexts. Current research projects expand the existing knowledge on the prevalence of sexual violence, present new prevention concepts, clarify the qualification needs of teachers, and point out challenges and opportunities in the development of institutional protection policies. However, new desiderata are also becoming apparent.
\end{abstract}

Keywords: sexual violence, school, prevention, intervention

\section{Einleitung}

Das Thema „Sexualisierte Gewalt“ wird im Kontext des Schulsystems gegenwärtig in durchaus ambivalenter Weise angesprochen. So verfolgt einerseits die Initiative des Unabhängigen Beauftragten für Fragen des sexuellen Kindesmissbrauchs (UBSKM) Schule gegen sexuelle Gewalt das Ziel, sämtliche Schulen in Deutschland dazu zu aktivieren, „Präventions- und Hilfemaßnahmen gegen sexuelle Gewalt zu entwickeln 
und Schutzkonzepte zum gelebten Schulalltag werden zu lassen“ (Rörig, 2017, S. 60). Die Initiative beruft sich darauf, dass nur in Schulen die Gesamtheit aller Kinder und Jugendlichen erreicht werden könne.

Demgegenüber stellt Poelchau (2018) die kritische, provokante und zugleich besorgte Frage, ob nicht gerade die zunehmende Beschäftigung mit Fragen von Aufklärung, Prävention und Intervention während der letzten Jahre sowie die sukzessive Implementierung entsprechender Strukturen und Maßnahmen in pädagogischen Einrichtungen dazu geführt habe, dass dieses „Thema durch“ und „für die Schule ausgeräumt" sei (ebd., S. 49). Somit scheint die durchaus streitbare Frage: „Was geht das die Schule an?" (Wanzeck-Sielert, 2010, S. 8), welche bereits zu Beginn der aufkeimenden Debatte aufgeworfen wurde, brisanter denn je zu sein.

Angesichts dieses Spannungsfeldes zwischen gesteuerter Aktivierung und mutmaßlich abflauendem Interesse, das gleichermaßen die Institution Schule und die Lehrerprofession als Ganzes, die einzelne Schule als Organisation sowie letztlich jede einzelne Lehrkraft betrifft, besteht Orientierungsbedarf. Als hilfreich erweist sich dabei eine Reflexion der aktuellen wissenschaftlichen Entwicklungen zum Verhältnis von Schule und sexualisierter Gewalt. Die folgende Auseinandersetzung mit der empirischen Forschung, ihren Konzepten und Befunden, Problemen und Desideraten möchte einen Beitrag zur notwendigen Standortbestimmung von Schule im Themenund Problemfeld von sexualisierter Gewalt leisten.

Prägend für die Entwicklung des Forschungsgeschehens war und ist weiterhin insbesondere die ab 2011 vom Bundesministerium für Bildung und Forschung (BMBF) getragene Förderlinie Sexuelle Gewalt gegen Kinder und Jugendliche in pädagogischen Kontexten. Diese erste einschlägige, bildungswissenschaftlich ausgerichtete Förderlinie hat bundesweit 17 Forschungsvorhaben, vier Juniorprofessuren und eine Forschungsprofessur unterstützt. Die geförderten Projekte befassen sich mit Fragen der Verbesserung des Schutzes von Kindern und Jugendlichen vor sexualisierter Gewalt in pädagogischen Einrichtungen. Den Professuren kommt überdies die Aufgabe einer nachhaltigen Etablierung des Themas an den Hochschulen in Forschung und Lehre zu (vgl. BMBF, 2016, S. 7). Expliziten Bezug zum Bereich Schule bzw. zur Lehrerprofessionalität weisen sechs der Projekte und drei der Professuren auf. Außer den auf eine Laufzeit von sechs Jahren angelegten Juniorprofessuren haben die Forschungsprojekte mittlerweile (weitgehend) ihren Abschluss gefunden und ihre Ergebnisse publiziert (für eine Gesamtdarstellung der Förderlinie vgl. BMBF, 2016).

Inwiefern nun die erklärte Zielsetzung dieser Forschungsförderung, eine neue „thematische Wissenschaftslandschaft" (ebd., S. 7) hervorzubringen, auch neue Impulse 
für die schulische Auseinandersetzung mit sexualisierter Gewalt geben kann, soll im Folgenden diskutiert werden. ${ }^{1}$

\section{Schule und sexualisierte Gewalt im Forschungsfokus}

Für die Forschung zu sexualisierter Gewalt gegen Kinder und Jugendliche markiert der Runde Tisch Sexueller Kindesmissbrauch in Abhängigkeits- und Machtverhältnissen in privaten und öffentlichen Einrichtungen und im familiären Bereich (2010-2011) eine Zäsur. Einberufen wurde er als Reaktion auf die Debatte um die u.a. am Berliner Canisius-Kolleg und an der hessischen Odenwaldschule aufgedeckten Übergriffe durch pädagogische bzw. geistliche Fach- und Leitungskräfte. Der schulische Kontext dieser Fälle spiegelt sich sowohl in der Besetzung des Runden Tisches mit zahlreichen Angehörigen des Bildungssystems als auch in dessen Abschlussbericht wider. Dort wird die besondere Rolle von Schule als „Schutzraum“ und Instanz für Aufklärung und Prävention betont (RTKM, 2011, S. 28).

Für die individuelle Ausgestaltung dieser Rolle seien Schulleitungen und Lehrkräfte zu qualifizieren und zu sensibilisieren (vgl. ebd., S. 191). Mit Blick auf die offengelegten Gewaltfälle werden Fragen nach dem Zustandekommen von sexualisierter Gewalt an Schulen sowie nach geeigneten Präventions- und Interventionsansätzen gestellt (vgl. ebd., S. 44). Die Forschungsförderung des BMBF bezieht sich in ihrer Ausrichtung explizit auf diese Desiderate (vgl. BMBF, 2016, S. 5).

Der Fokus, der sich infolgedessen auf Schulen richtet, weist eine neue Qualität und Intensität auf, knüpft aber auch an bestehende Forschungsbefunde an. Schubarth \& Ulbricht (2012) konstatieren für den älteren Fachdiskurs, dass er zwar immer wieder auch empirische Hinweise $\mathrm{zu}$ sexualisierter Gewalt hervorgebracht habe, andere Gewaltphänomene wie z.B. Bullying jedoch sehr viel ausführlicher thematisiere. Zum gleichen Schluss kommt Kindler (2014a, S. 121) mit Blick auf die internationale Forschung. Als aktuelles Beispiel für die auch international zunehmende Aufmerksamkeit kann die von der Australischen Royal Commission into Institutional Responses to Child Sexual Abuse angeregte Forschung dienen. Auch hier wird vor dem Hintergrund zahlreicher institutioneller Missbrauchsfälle die Auseinandersetzung von Schulen mit ihrer Verantwortung für den Schutz vor sexualisierter Gewalt als unabdingbar charakterisiert (vgl. Palmer \& Feldman, 2017).

Die fachliche Zurückhaltung verwundert nicht zuletzt angesichts des Konsenses, dass jegliche Missbrauchserfahrung, über alle anderen schädigenden Folgen hinaus,

1 Der Beitrag entstammt dem von der Juniorprofessur „Pädagogische Professionalität gegen sexuelle Gewalt - Prävention, Kooperation, Intervention“ durchgeführten Teilprojekt des ebenfalls BMBF-geförderten Meta-Vorhabens „Profilbildung, Praxistransfer, Ethik und Monitoring" (ProPEM) in der Förderlinie Sexuelle Gewalt in pädagogischen Kontexten. 
eine erhebliche negative Wirkung auf die Schulleistung ausüben kann. Glammeier \& Fein (2018) mutmaßen, dass die Annahme sexueller Gewalterfahrung als mögliche Ursache für Leistungseinbrüche „noch nicht unbedingt im Wahrnehmungshorizont von Lehrkräften enthalten“ sei (ebd., S. 691).

Daneben dürfte es auch von Bedeutung sein, dass der Forschungszugang zu Schulen mit erheblichen, sowohl formellen als auch forschungsethischen, Herausforderungen verbunden ist. Exemplarisch sei auf die von der Universität Regensburg angestrebte Schülerbefragung im Rahmen der MiKADO-Studie (Missbrauch von Kindern: Aetiologie, Dunkelfeld, Opfer) hingewiesen, die nach vehementen Elternprotesten und großer öffentlicher Aufmerksamkeit von mehreren Kultusministerien keine Durchführungsgenehmigung erhalten hatte (vgl. Jud, Rassenhofer, Witt, Münzer \& Fegert, 2016, S. 33). Entzündet hatten sich die Proteste an expliziten Fragen zu sexualisierten Gewalthandlungen in den MiKADO-Fragebögen.

Gleichwohl gehen Schubarth \& Ulbricht (2012, S. 246) davon aus, dass die Befundlage schon vor dem Einsetzen neuer Forschungsbemühungen hinreichend aussagekräftig gewesen sei, um sexualisierte Übergriffe im schulischen Kontext als Problem anzuerkennen. Vor allem in unterschiedlichen Studien während der 1990er-Jahre konnten bereits aussagekräftige Befunde zur Prävalenz von sexualisierter Gewalt zwischen Schüler*innen gewonnen werden. Deutlich wurde dabei auch eine eher gering ausgeprägte schulische Interventionsbereitschaft (Forschungsgruppe Schulevaluation, 1998). Auch sexuell übergriffiges Lehrerhandeln wurde überwiegend im Kontext von allgemeinen pädagogischen Grenzüberschreitungen aufgegriffen (vgl. Schubarth \& Ulbricht, 2012, S. 243). Von Bedeutung sind diesbezüglich insbesondere die Studien aus dem INTAKT-Projektnetz (Soziale Interaktionen in pädagogischen Arbeitsfeldern), die u. a. missachtendes Lehrerhandeln im Unterricht in den Blick nehmen (Prengel, 2012).

Vor dem Hintergrund der jüngeren Debatte um sexualisierte Gewalt in pädagogischen Institutionen haben nun Überlegungen an Relevanz gewonnen, die betonen, dass Schule nicht per se Schutzraum sei, sondern spezifische Opportunitätsstrukturen für die Ausübung sexualisierter Gewalt aufweise. So zeigen Helsper \& Reh (2012) auf, wie beispielsweise dysfunktionale Gestaltungen der Antinomie von Nähe und Distanz „schulkulturelle Möglichkeitsräume“ für sexualisierter Gewalt erzeugen (ebd., S. 278). Sexualisierte Gewalt in Schule wird demnach als Resultat systemischer Bedingungsgefüge und -strukturen angesehen (vgl. Kindler, 2014a, S. 123).

Die Institutionenbefragung des Deutschen Jugendinstituts (DJI) (Helming et al., 2011) konnte zur aktuellen Prävalenz von sexualisierter Gewalt in Schule umfangreiche Daten liefern. Die befragten Schulleitungen und Lehrkräfte benannten zu über 40 Prozent (Verdachts-)Fälle von Übergriffen sowohl durch Schulpersonal als auch unter Schüler*innen, vor allem aber durch schulfremde Personen (vgl. ebd., S. 62). 
Es konnte in dieser Studie jedoch auch festgehalten werden, dass Lehrkräfte und Schulsozialarbeit wichtige Ansprechpersonen bei Offenlegungen („Disclosure“) von Gewalterfahrung sein können (vgl. ebd., S. 96).

Die Ergebnisse der zuletzt an hessischen Schulen durchgeführten SPEAK-Studie (Sexuelle Gewalt in der Erfahrung Jugendlicher-SPEAK) untermauern, dass insbesondere verbale, vielfach aber auch körperliche Formen sexualisierter Gewalt für zahlreiche Mädchen und Jungen fester Bestandteil schulischer Alltagserfahrung sind (Maschke \& Stecher, 2018). Böhm, Budde \& Dekker (2018) weisen schließlich auf die Problematik von sexualisierter Gewalt durch digitale Medien hin, die für Schulen als hochaktuell gilt, jedoch eher geringe Aufmerksamkeit erfährt.

\section{Zentrale Befunde und Implikationen ausgewählter Forschungsprojekte der BMBF-Förderlinie Sexuelle Gewalt gegen Kinder und Jugendliche in pädagogischen Kontexten}

Im Rahmen dieses Beitrags kann und soll keine vollständige Übersicht und systematische Zusammenfassung der schulbezogenen Forschungsergebnisse aus der BMBFFörderlinie geboten werden. Im Folgenden werden daher ausgewählte Schlüsselbefunde aus einzelnen Projekten vorgestellt, die für die Bereiche Grundlagenwissen, Handlungswissen und Anwendungsbezug als besonders aussagekräftig erachtet werden.

\subsection{Prävalenz sexualisierter Gewalt in Schule}

Die lückenhaften Daten zur Prävalenz von sexualisierter Gewalt im schulischen Bereich erfahren durch das DJI-Projekt Wissen von Schülerinnen und Schülern über sexuelle Gewalt in pädagogischen Kontexten substanzielle Ergänzung (Hofherr \& Kindler, 2018). Bestätigung finden die Annahmen zum insgesamt hohen Verbreitungsgrad von sexualisierter Gewalterfahrung. 66 Prozent der befragten Mädchen und 52 Prozent der Jungen geben mindesten eine erlebte Situation an (vgl. ebd., S. 100). Insbesondere die Häufigkeit verbaler Gewaltformen sticht hervor. Während verbale sexualisierte Gewalt größtenteils in der Schule stattfindet, geschehen körperliche Übergriffe zumeist andernorts (vgl. ebd., S. 101).

Hinsichtlich der Tatverantwortlichen fällt auf, dass überwiegend Gleichaltrige genannt werden. Sexualisierte Gewalt durch Schulpersonal kommt zwar vor, stellt aber eine Seltenheit dar. Erhellend sind ebenfalls die Ergebnisse zur Offenlegung von Gewalterfahrung gegenüber Schulpersonal. Hier zeigt sich, dass Mädchen sowie ältere Jugendliche sich vergleichsweise häufig an Lehrkräfte wenden, während 
Jungen und Mädchen mit Migrationshintergrund dies eher selten tun. Auch Fortbildungserfahrung von Lehrkräften scheint positiv mit der Offenlegungsbereitschaft zusammenzuhängen.

Irritierend ist hingegen der Befund, dass eine hohe wahrgenommene Interventionsbereitschaft von Lehrkräften sich negativ auf die Bereitschaft, ihnen einen Übergriff anzuvertrauen, auszuwirken scheint. Hier mutmaßen die Forscher, dass die Interventionen der Lehrkräfte nicht als ausreichend vertrauensstiftend eingestuft werden (vgl. ebd., S. 104).

\subsection{Ein Präventionsprogramm für Grundschulen}

Sehr dezidierten Anwendungsbezug weist das IGEL-Projekt (Entwicklung, Implementierung und Evaluation eines schulbasierten Gruppenprogramms zur Prävention von sexualisierter Gewalt in der Primarstufe) auf, in dessen Rahmen ein umfangreiches Präventionsprogramm für Grundschulen entwickelt wurde. Das Konzept ist als Manual publiziert, das neben einer detaillierten Beschreibung der Programmelemente auch Informationen und Grundlagen zu sexualisierter Gewalt, Täterstrategien, Intervention und Prävention enthält (Körner, Bauer \& Kreuz, 2016). Einzelne Lehrkräfte oder ganze Kollegien können sich auf diese Weise in Kombination mit einer zugehörigen Fortbildungseinheit für die Durchführung des Programms qualifizieren.

Das IGEL-Programm zeichnet sich dadurch aus, dass es auf einer Reflexion empirisch nachweisbarer Defizite von Präventionsmaßnahmen basiert und Evaluationsergebnisse zu seiner Umsetzung vorliegen. Es gibt dabei Hinweise auf signifikante Effekte einer zumindest kurz- und mittelfristigen Vergrößerung des altersgerechten Wissens der Teilnehmenden über sexualisierte Gewalt und mögliche Handlungsoptionen. Negative Auswirkungen wie erhöhte Ängstlichkeit werden hingegen nicht berichtet (vgl. ebd., S. 175). Wenngleich die Präventionsthematik in der Grundschule nicht grundsätzlich neu ist, stellt das IGEL-Programm aufgrund seiner fundierten Aufbereitung und konkreten Darbietung, die eine niedrigschwellige Umsetzbarkeit ermöglichen will, eine Innovation dar.

\subsection{Qualifizierung von Lehrkräften}

Eine Verschränkung von Grundlagenwissen und Anwendungsbezug findet sich im Kontext des Projektes Sexualisierte Übergriffe und Schule - Prävention und Intervention. In der hier durchgeführten Lehrkräftebefragung geben 26 Prozent der Teilnehmenden an, mit Vor- oder Verdachtsfällen konfrontiert worden zu sein (vgl. Glammeier, 2015, S. 14). Darauf bezugnehmend werden Maximen beschrieben, die Lehrkräften im Umgang mit (mutmaßlich) betroffenen Kindern und Jugendlichen 
Orientierung bieten können. Hierzu zählen etwa Parteilichkeit und Solidarität sowie eine adäquate Gesprächsführung. Die hier dokumentierte Rolle von Lehrkräften als Ansprechpersonen steht jedoch im Kontrast $\mathrm{zu}$ Befunden der qualitativen Zugänge dieses Projekts. Interviews mit Lehrkräften lassen Rückschlüsse auf ausgeprägte Tendenzen zu, Schilderungen von sexualisierter Gewalterfahrung für unwahr oder zumindest übertrieben zu halten. Dafür scheinen insbesondere fehlerhafte und mythenbasierte Vorstellungen von sexualisierter Gewalt in Verbindung mit Geschlechtsstereotypen ursächlich zu sein (vgl. Glammeier \& Fein, 2018, S. 693).

Es wird auch deswegen die Bedeutung von Schutzkonzepten als Teil von Schulentwicklungsprozessen betont. Konkret wird auf die Erarbeitung von Verhaltenskodizes, Handlungsplänen und Beschwerdesystemen, die Durchführung von Risikoanalysen sowie Kooperation mit externen Fachkräften hingewiesen (vgl. Glammeier, 2015, S. 15). Auch aus den im Rahmen des Projekts durchgeführten Lehrerfortbildungen können Wissensdefizite bzw. Fortbildungsbedarfe ermittelt werden (vgl. Glammeier \& Fein, 2018, S. 690). Hierzu zählen insbesondere Wissen über Kindeswohlgefährdung, Qualifizierung im Bereich der Krisenkommunikation und reflexive Kompetenz im Umgang mit eigenen Affekten. Die Projekttätigkeit mündete in die Entwicklung eines neuartigen Fortbildungskonzeptes. Dieses sieht intensive Schulungsmaßnahmen für einzelne Lehrkräfte und Schulleitungen vor sowie eine engmaschige Begleitung durch externe Fachberatung. Durch kombinierte und bereichsspezifische Qualifizierung soll eine dynamische Schutzkonzeptentwicklung mit festen personellen Zuständigkeiten und klaren Handlungsabläufen bei Verdachtsfällen ermöglicht werden; gleichzeitig wird auf die Notwendigkeit einer bundesweiten Fortbildungsoffensive für Schulen hingewiesen (vgl. ebd., S. 697).

Ergänzen lassen sich Befunde aus dem SeMB-Projekt (Sexueller Missbrauch an Kindern und Jugendlichen mit Behinderung), in dem u. a. Fortbildungen mit Förderschullehrkräften im Beruf und im Studium durchgeführt wurden. Die quantitativstatistische Evaluation dieser Fortbildungsmaßnahmen belegt signifikant positive Effekte im Bereich des deklarativen Wissens über sexualisierte Gewalt, eine positive Beeinflussung der Einstellung zu Prävention, jedoch keine Verringerung hemmender Gefühle im Umgang mit Verdachtsfällen (Scharmanski, Urbann, Verlinden \& Bienstein, 2015).

Die Thematik der Qualifizierung erstreckt sich nicht nur auf die Bereiche deklarativen und prozeduralen Wissens, sondern wird auch als Element reflexiver Professionalität begriffen. So markieren die Befunde der berufsbiographischen Forschung der Juniorprofessur in Kassel den Umgang mit Verdachtsfällen auf sexualisierte Gewalt als besonders reflexionsbedürftig (Retkowski, Dekker, Henningsen, Voß \& Wazlawik, 2018). Subjektiv geprägte Vorstellungen von Sexualmoral, berufsbiographische Prägungen und die Struktur der Lehrkraft-Schüler-Beziehung seien demnach einem Prozess der schulöffentlichen Auseinandersetzung zugänglich zu machen. Auf diese Weise sei 
der nach wie vor tabuisierte Umgang mit schwebenden Verdachtsfällen mit neuen Impulsen zu versehen. Gleichzeitig wird eine „sektorale Trennung von professionellen/organisationalen und ethischen Fragen" befürwortet (ebd., S. 169). Es bestehe die Notwendigkeit, unterschiedliche Akteure wie Elternräte und multiprofessionelle pädagogische Ethik-Komitees im schulischen Bereich in Verantwortung zu bringen.

Eine noch laufende Entwicklung zur systematischen Verankerung einer grundständigen Qualifizierung in den pädagogischen Studiengängen stellt das von den Juniorprofessuren und der Forschungsprofessur erarbeitete Basis-Curriculum Grundlagen sexualisierter Gewalt in pädagogischen Institutionen dar (Retkowski et al., 2018). In drei kohärenten Seminarveranstaltungen werden reflexive Zugänge zu den Themenbereichen "Sexualisierte Gewalt in pädagogischen Einrichtungen“, „Sexuelle Sozialisation" und "Sexuelle Bildung" sowie „Professionalität und Ethik“ eröffnet. Bis 2019 wird dieses Curriculum durch eine Projektförderung des Bundesministeriums für Familien, Senioren, Frauen und Jugend (BMFSFJ) an den Standorten Hamburg und Münster evaluiert, um darauf aufbauend ein hochschuldidaktisches Konzept zu entwickeln.

\subsection{Institutionelle Schutzkonzepte}

Die vielversprechenden Belege dafür, dass Qualifizierungsmaßnahmen messbare Wirkungen erzielen können, sind jedoch den Ergebnissen des Projektes Ich bin sicher gegenüberzustellen. Demnach erleben sich Fortbildungsteilnehmende mit ihrem neu erworbenen Wissen in ihren Organisationen häufig als isoliert und nehmen wenig Spielraum für organisationale Veränderungen wahr (vgl. Fegert, Schröer \& Wolff, 2017, S. 243).

Herausforderungen bei präventionsorientierter Schulentwicklung ermitteln auch Schwerdt und Wazlawik (2017) im Forschungsprojekt Institutionelle Schutzkonzepte in der Schule. Dazu zählen dem normierenden Anspruch von Schutzkonzepten geschuldete Spannungsfelder. Sehr allgemeine normative Formulierungen, etwa zur generellen Wichtigkeit eines achtsamen Umgangs, stehen hochgradig detaillierten Handlungsanweisungen gegenüber, was Probleme für die Umsetzbarkeit in pädagogische Praxis nach sich zieht. Dies betrifft beispielsweise die konkrete situative Gestaltung von Nähe und Distanz unter Beachtung entsprechender Vorgaben. Sowohl zu allgemeine als auch zu konkrete Handlungsnormen können Irritationen evozieren, die mithin die Bereitschaft der Fachkräfte zur Orientierung an einem Schutzkonzept beeinträchtigen. Auch organisatorische und logistische Rahmenbedingungen von Schutzkonzeptentwicklung werden als voraussetzungsreich beschrieben, da die zugehörigen Arbeitsprozesse ressourcenintensiv und von wechselvollen sozialen Dynamiken begleitet seien (vgl. ebd., S. 68). 
Zusammenfassend lässt sich sagen, dass die im Abschlussbericht des Runden Tisches mit Blick auf schulische Kontexte benannten Forschungsdesiderate in den Befunden der Förderlinienprojekte vielfache Entsprechungen finden. Sowohl das Grundlagenwissen zu sexualisierter Gewalt im Bereich Schule als auch das Wissen zum Qualifizierungsbedarf von Lehrkräften und zu entsprechenden Fortbildungsmöglichkeiten wird signifikant erweitert. Auf diese Weise werden Relevanz und Aktualität der Problemdimension und des Handlungsbedarfs unterstrichen. Damit einher gehen jedoch auch die Formulierung neuer Forschungsfragen sowie Fragen nach der Anschlussfähigkeit der Forschungsbefunde an schulische Strukturen.

\section{Bilanz und Ausblick}

Die Befunde der BMBF-geförderten Forschungsprojekte mit Schulbezug decken ein breites Spektrum an Fragestellungen und Themen ab und zeitigen vielversprechende Ergebnisse. Dennoch ist festzuhalten, dass beispielsweise die Heterogenität des Schulsystems nur eingeschränkt abgebildet ist und auch eine systematisch angelegte Vergleichsperspektive auf die einzelnen Bundesländer fehlt. Mit Fragen verbunden bleibt die Implementierung von Schutzkonzepten, insbesondere wie hierdurch nachvollziehbare Veränderungen von Schulklima und Schulkultur entstehen können. Dies umfasst ebenso die Evaluation einzelner Maßnahmen wie die schulbasierter Präventionsprogramme oder Beschwerdesysteme sowie deren Zusammenwirken.

Aber auch weitere genuin schulische Elemente wie die Sexualerziehung sind in ihrem Verhältnis zu sexualisierter Gewalt kaum untersucht. Dies betrifft auch die präventive und protektive Rolle spezialisierter Fachkräfte, z.B. Beratungs- oder Vertrauenslehrkräfte, Schulsozialarbeiter*innen oder Präventionsbeauftragte. Auch über multiprofessionelle Kooperationsprozesse zwischen schulinternen und -externen Fachkräften existiert kaum empirisch abgesichertes Wissen. Die vorhandene Forschung legt zudem nahe, in künftigen Studien besonderes Augenmerk auf das Verhältnis nomineller Schutzstrukturen und alltäglicher schulischer Konditionen präventiven und intervenierenden Handelns zu richten. Dies lässt sich mit Kindler (2014b, S. 87) dadurch begründen, dass „gelebte Normen hierbei einflussreicher sind als formulierte Normen“.

Trotz fortbestehender und neu hinzugekommener Forschungsdesiderate hat die Forschungstätigkeit im Rahmen der BMBF-Förderlinie einen wichtigen Beitrag dazu geleistet, Forschungslücken zu verkleinern, blinde Flecken auszuleuchten und die gesamte Thematik deutlicher zu konturieren. Nicht zuletzt für die ethische Fundierung von Forschungsvorhaben unter Beteiligung von Kindern und Jugendlichen hat das Wissenschaftsnetzwerk wichtige Impulse hervorgebracht. Mit der „Bonner EthikErklärung“ wurde der erste einschlägige (deutschsprachige) Ethikkodex veröffentlicht 
(Poelchau, Briken, Wazlawik, Bauer, Fegert \& Kavemann, 2015). Die Auseinandersetzung mit forschungsethischen Herausforderungen stellt gerade in schulbezogener Forschung einen konstitutiven Anspruch dar, wie Maschke \& Stecher (2018, S. 101) zuletzt vor dem Hintergrund der SPEAK-Studie aufzeigen. Es werden daher insbesondere Möglichkeiten diskutiert, Forschungsansätze umfassend partizipativ zu konzipieren (Schlingmann, 2015).

Für die vorgestellten Forschungsbefunde ist die Frage nach einer konkreten Umsetzung oder Anwendung in schulpädagogischer Praxis gegenwärtig noch überwiegend unbeantwortet. Während die Publikation von Ergebnissen zwar umfangreich erfolgt, fehlen derzeit noch Belege, wie und in welchem Umfang sie nachvollziehbar Eingang in Schulentwicklungsprozesse, Schutzkonzepte oder Aus- und Fortbildungsmaßnahmen finden. Die aktualisierte Befundlage zu Schule und sexualisierter Gewalt bietet somit insgesamt für die weitere Gestaltung von Prozessen des Wissenschaft-Praxis-Transfers und die Planung neuer Forschungsvorhaben zahlreiche Anschlussmöglichkeiten. Insbesondere die Berücksichtigung partizipativer Elemente ist dabei als Herausforderung, aber auch als Chance für kreative Herangehensweisen zu werten.

Von Bedeutung für die Planung weiterführender Forschungsvorhaben ist es überdies, aktuelle Schulthemen und -entwicklungen zu berücksichtigen, etwa im Zusammenhang mit Inklusion oder der Ausweitung von Ganztagsangeboten. Wird die Prävention von sexualisierter Gewalt hier nicht als Querschnittsthema aufgegriffen und kontinuierlich mitgedacht, droht ihr in der Tat ein neuerlicher Bedeutungs- und Aktualitätsverlust.

\section{Literatur und Internetquellen}

BMBF (Bundesministerium für Bildung und Forschung) (Hrsg.). (2016). Sexuelle Gewalt gegen Kinder und Jugendliche in pädagogischen Kontexten. Forschung zu Prävention und Schutzkonzepten. Berlin: BMBF.

Böhm, M., Budde, J., \& Dekker, A. (2018). Sexuelle Grenzverletzungen mittels digitaler Medien an Schulen - Annäherung an einen doppelten Verdeckungszusammenhang. MedienPädagogik: Zeitschrift für Theorie und Praxis der Medienbildung, Einzelbeiträge, $1-18$.

Fegert, J. M., Schröer, W., \& Wolff, M. (2017). Schutzkonzepte im Transfer. Übersetzungsprozesse zwischen Forschung und Organisationsentwicklung. In M. Wolff, W. Schröer \& J. M. Fegert (Hrsg.), Schutzkonzepte in Theorie und Praxis. Ein beteiligungsorientiertes Werkbuch (S. 238-244). Weinheim: Beltz Juventa.

Forschungsgruppe Schulevaluation (Hrsg.). (1998). Gewalt als soziales Problem in Schulen. Die Dresdner Studie. Untersuchungsergebnisse und Präventionsstrategien. Opladen: Leske + Budrich. doi.org/10.1007/978-3-322-92271-7

Glammeier, S. (2015). Pädagogische Herausforderungen angesichts sexueller Gewalt - was Schulen und Lehrkräfte tun können. Die berufsbildende Schule, 67 (1), 13-17. 
Glammeier, S., \& Fein, S. (2018). Sexualisierte Gewalt als Thema in der Aus- und Fortbildung in Schulen. In A. Retkowski, A. Treibel \& E. Tuider (Hrsg.), Handbuch Sexualisierte Gewalt und pädagogische Kontexte. Theorie, Forschung, Praxis (S. 689699). Weinheim: Beltz Juventa.

Helming, E., Kindler, H., Langmeyer, A., Mayer, M., Mosser, P., Entleitner, C., Schutter, S., \& Wolff, M. (2011). Sexuelle Gewalt gegen Mädchen und Jungen in Institutionen. Abschlussbericht des DJI-Projekts. München: Deutsches Jugendinstitut e.V., Abteilung Familie und Familienpolitik.

Helsper, W., \& Reh, S. (2012). Nähe, Diffusität und Asymmetrie in pädagogischen Interaktionen. In W. Thole, M. S. Baader, W. Helsper, M. Kappeler, M. LeuzingerBohleber, S. Reh et al. (Hrsg.), Sexualisierte Gewalt, Macht und Pädagogik (S. 265-290). Opladen: Barbara Budrich.

Hofherr, S., \& Kindler, H. (2018). Sexuelle Übergriffe in Schulen aus der Sicht von Schülerinnen und Schülern. Zusammenhänge zum Erleben von Schule und der Bereitschaft zur Hilfesuche. In S. Andresen \& R. Tippelt (Hrsg.), Sexuelle Gewalt in Kindheit und Jugend. Theoretische, empirische und konzeptionelle Erkenntnisse und Herausforderungen erziehungswissenschaftlicher Forschung (Zeitschrift für Pädagogik, 64. Beiheft) (S. 95-110). Weinheim: Beltz Juventa.

Jud, A., Rassenhofer, M., Witt, A., Münzer, A., \& Fegert, J. M. (2016). Häufigkeitsangaben zum sexuellen Missbrauch. Internationale Einordnung, Bewertung der Kenntnislage in Deutschland, Beschreibung des Entwicklungsbedarfs. Hrsg. vom Unabhängigen Beauftragten für Fragen des sexuellen Kindesmissbrauchs. Berlin: UBSKM.

Kindler, H. (2014a). Sexuelle Übergriffe in Schulen. In H. Willems \& D. Ferring (Hrsg.), Macht und Missbrauch in Institutionen. Interdisziplinäre Perspektiven auf institutionelle Kontexte und Strategien der Prävention (S. 111-131). Wiesbaden: Springer VS. doi. org/10.1007/978-3-658-04297-4_7

Kindler, H. (2014b). Wirkungen, Nebenwirkungen und ungelöste Probleme bei der Prävention von sexueller Gewalt gegen Kinder und Jugendliche. In K. Böllert \& M. Wazlawik (Hrsg.), Sexualisierte Gewalt. Institutionelle und professionelle Herausforderungen (S. 77-94). Wiesbaden: Springer VS. doi.org/10.1007/978-3-531-19095-2_7

Körner, W., Bauer, U., \& Kreuz, I. (Hrsg.). (2016). Prävention von sexualisierter Gewalt in der Primarstufe. Manual für Lehrerinnen und Lehrer. Das IGEL Programm. Weinheim: Beltz Juventa.

Maschke, S., \& Stecher, L. (2018). Sexuelle Gewalt: Erfahrungen Jugendlicher heute. Weinheim: Beltz Juventa.

Palmer, D., \& Feldman, V. (2017). Toward a More Comprehensive Analysis of the Role of Organizational Culture in Child Sexual Abuse in Institutional Contexts. Child Abuse \& Neglect, 74, 23-34. doi.org/10.1016/j.chiabu.2017.08.004

Poelchau, H.-W. (2018). Editorial: Sexueller Missbrauch und Schule - Thema durch? Schulverwaltung spezial: Zeitschrift für Schulgestaltung und Schulentwicklung, 20 (2), 49.

Poelchau, H.-W., Briken, P., Wazlawik, M., Bauer, U., Fegert, J. M., \& Kavemann, B. (2015). Bonner Ethik-Erklärung. Zeitschrift für Sexualforschung, 28 (2), 153-160. doi. org/10.1055/s-0035-1553220

Prengel, A. (2012). Projektnetz „INTAKT“ (Soziale Interaktionen in pädagogischen Arbeitsfeldern). In A. Prengel \& H. Schmitt (Hrsg.), Netzpublikationen des Arbeitskreises Menschenrechtsbildung in der Rochow-Akademie für historische und zeitdiagnostische Forschung an der Universität Potsdam. Zugriff am 15.01.2019. Verfügbar unter: http:// www.rochow-museum.uni-potsdam.de/arbeitskreis-menschenrechtsbildung/netzpubli kationen-des-ak-mrb.html.

Retkowski, A. (2018). Professionelle, organisationale und schulöffentliche Selbstverständigungsprozesse im Kontext schwebender Verdachtsfälle auf sexualisierte Gewalt. In 
S. Andresen \& R. Tippelt (Hrsg.), Sexuelle Gewalt in Kindheit und Jugend. Theoretische, empirische und konzeptionelle Erkenntnisse und Herausforderungen erziehungswissenschaftlicher Forschung (Zeitschrift für Pädagogik, 64. Beiheft) (S. 162-171). Weinheim: Beltz Juventa.

Retkowski, A., Dekker, A., Henningsen, A., Voß, H.-J., \& Wazlawik, M. (2018). BasisCurriculum zur Verankerung des Themas "Sexuelle Gewalt in Institutionen“ in universitärer und hochschulischer Lehre. In M. Wazlawik, H.-J. Voß, A. Retkowski, A. Henningsen, \& A. Dekker (Hrsg.), Sexuelle Gewalt in pädagogischen Kontexten. Aktuelle Forschungen und Reflexionen (S. 261-289). Wiesbaden: Springer VS.

Rörig, J.-W. (2017). Schweigen hilft Tätern und Täterinnen. Was für einen verbesserten Schutz vor sexuellem Missbrauch getan werden kann. Blätter der Wohlfahrtspflege, 164 (2), 59-62. doi.org/10.5771/0340-8574-2017-2-59

RTKM (2011). Runder Tisch Sexueller Kindesmissbrauch in Abhängigkeits- und Machtverhältnissen in privaten und öffentlichen Einrichtungen und im familiären Bereich Abschlussbericht. Hrsg. vom Bundesministerium der Justiz, vom Bundesministerium für Familie, Senioren, Frauen und Jugend \& vom Bundesministerium für Bildung und Forschung. Berlin: BMJ, BMFSF \& BMBF.

Scharmanski, S., Urbann, K., Verlinden, K., \& Bienstein, P. (2015). Sexueller Missbrauch an Kindern und Jugendlichen mit Hörbehinderung - Teil II. Inhalte und Ergebnisse der SeMB-Fortbildung für Lehrer/innen an Schulen mit dem Förderschwerpunkt Hören und Kommunikation. Das Zeichen: Zeitschrift für Sprache und Kultur Gehörloser, 29 (101), 380-391.

Schlingmann, T. (2015). Für ein neues Verhältnis von Wissenschaft, Praxis und Betroffenen. Anmerkungen aus der Perspektive eines forschenden, betroffenen Praktikers. Zeitschrift für Sexualforschung, 28 (4), 349-362.

Schubarth, W., \& Ulbricht, J. (2012). Sexualisierte Gewalt an Schulen. In W. Thole, M. S. Baader, W. Helsper, M. Kappeler, M. Leuzinger-Bohleber, S. Reh et al. (Hrsg.), Sexualisierte Gewalt, Macht und Pädagogik (S. 238-248). Opladen: Barbara Budrich.

Schwerdt, D., \& Wazlawik, M. (2017). Institutionelle Schutzkonzepte in der Schule. Kinderund Jugendschutz in Wissenschaft und Praxis, 62 (2), 66-70.

Wanzeck-Sielert, C. (2010). „Was geht das die Schule an?““ Zur Handlungskompetenz von Lehrkräften, Schulleitungen und Schulen bei sexueller Gewalt. Lernende Schule, 13 (51), 8-11.

Bernd Christmann, M. A., geb. 1979, Wissenschaftlicher Mitarbeiter in der Arbeitsgruppe „Pädagogische Professionalität gegen sexuelle Gewalt - Prävention, Kooperation, Intervention“ am Institut für Erziehungswissenschaft der Westfälischen Wilhelms-Universität Münster.

E-Mail: bernd.christmann@wwu.de

Daniel Schwerdt, M. A., geb. 1989, Wissenschaftlicher Mitarbeiter in der Arbeitsgruppe „Pädagogische Professionalität gegen sexuelle Gewalt - Prävention, Kooperation, Intervention“ am Institut für Erziehungswissenschaft der Westfälischen Wilhelms-Universität Münster.

E-Mail: daniel.schwerdt@wwu.de 
Martin Wazlawik, Jun.-Prof. Dr., geb. 1982, Juniorprofessor für „Pädagogische Professionalität gegen sexuelle Gewalt - Prävention, Kooperation, Intervention“ am Institut für Erziehungswissenschaft der Westfälischen Wilhelms-Universität Münster.

E-Mail: martin.wazlawik@wwu.de

Anschrift: Westfälische Wilhelms-Universität Münster, Institut für Erziehungswissenschaft, Georgskommende 33, 48143 Münster

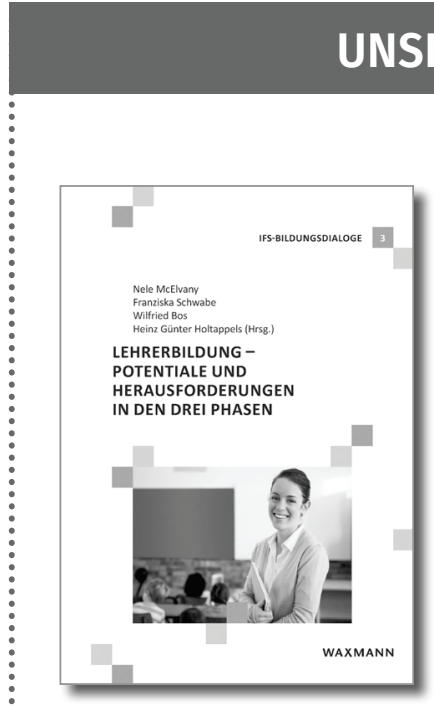

IFS-Bildungsdialoge, Band 3, 2019, 134 Seiten, br., 27,90€, ISBN 978-3-8309-3966-5

E-Book: 24,99€, ISBN 978-3-8309-8966-0
Nele McElvany, Franziska Schwabe, Wilfried Bos, Heinz Günter Holtappels (Hrsg.)

\section{Lehrerbildung -} Potentiale und Herausforderungen in den drei Phasen

Eine Lehrerbildung, die Lehrkräfte befähigt, Lern- und Bildungsprozesse erfolgreich anzuregen und zu gestalten, ist auch mit Blick auf aktuelle Entwicklungen wie der zunehmenden Inklusion oder der Integration neu zugewanderter Schülerinnen und Schüler eine der zentralen Herausforderungen des deutschen Bildungssystems. Die Lehrerbildung bildet die Basis der alltäglichen Arbeit von Lehrpersonen und ist einerseits mit großen Chancen wie der systematischen Verbesserung der Unterrichtsqualität über Schulstufen hinweg verbunden. Andererseits gibt es substantielle Herausforderungen, z.B. durch die zunehmende Digitalisierung. Dieser Band versammelt Perspektiven aus Bildungsforschung, -praxis, -politik und -verwaltung mit dem Ziel, erfolgreiche Strategien und Konzepte sowie zukünftige Bedarfe und Desiderate für eine gelingende Lehrerbildung zu identifizieren und zu diskutieren. 